\title{
Coronary artery calcification and epicardial adipose tissue as independent predictors of mortality in COVID-19
}

\author{
Leandro Slipczuk ${ }^{1,2}$ (1) $\cdot$ Francesco Castagna ${ }^{1} \cdot$ Alison Schonberger $^{3} \cdot$ Eitan Novogrodsky $^{3} \cdot$ Richard Sekerak $^{2}$. \\ Damini Dey ${ }^{4}$. Ulrich P. Jorde ${ }^{1,2}$. Jeffrey M. Levsky ${ }^{2,3} \cdot$ Mario J. Garcia ${ }^{1,2,3}$
}

Received: 1 March 2021 / Accepted: 3 May 2021 / Published online: 12 May 2021

(c) The Author(s), under exclusive licence to Springer Nature B.V. 2021

\begin{abstract}
Recent epidemiological studies have demonstrated that common cardiovascular risk factors are strongly associated with adverse outcomes in COVID-19. Coronary artery calcium (CAC) and epicardial fat (EAT) have shown to outperform traditional risk factors in predicting cardiovascular events in the general population. We aim to determine if CAC and EAT determined by Computed Tomographic (CT) scanning can predict all-cause mortality in patients admitted with COVID-19 disease. We performed a retrospective, post-hoc analysis of all patients admitted to Montefiore Medical Center with a confirmed COVID-19 diagnosis from March 1st, 2020 to May 2nd, 2020 who had a non-contrast CT of the chest within 5 years prior to admission. We determined ordinal CAC scores and quantified the epicardial (EAT) and thoracic (TAT) fat volume and examined their relationship with inpatient mortality. A total of 493 patients were analyzed. There were 197 deaths (39.95\%). Patients who died during the index admission had higher age (72, [64-80] vs 68, [57-76]; $\mathrm{p}<0.001)$, CAC score $(3,[0-6]$ vs $1,[0-4] ; p<0.001)$ and EAT $(107,[70-152]$ vs $94,[64-129] ; p=0.023)$. On a competing risk analysis regression model, CAC $\geq 4$ and EAT $\geq$ median $(98 \mathrm{ml})$ were independent predictors of mortality with increased mortality of $63 \%$ $(p=0.003)$ and $43 \%(p=0.032)$, respectively. As a composite, the group with a combination of CAC $\geq 4$ and EAT $\geq 98 \mathrm{ml}$ had the highest mortality. CAC and EAT measured from chest CT are strong independent predictors of inpatient mortality from COVID-19 in this high-risk cohort.
\end{abstract}

Keywords Covid $\cdot$ Coronary artery calcium $\cdot$ Epicardial adipose tissue $\cdot$ Mortality

\section{Introduction}

Coronavirus disease (COVID-19), caused by the severe acute respiratory syndrome coronavirus 2 (SARS-CoV-2) has rapidly spread globally. Although most cases recover, COVID-19 still appears to retain an elevated mortality with the number of deaths escalating to over 1.5 million worldwide through December 2020 [1]. COVID-19 is associated with a high incidence of thrombotic complications. It has

Leandro Slipczuk

lslipczukb@montefiore.org

1 Cardiology Division, Montefiore Medical Center, $111 \mathrm{E}$ 210th, Bronx, NY 10467, USA

2 Albert Einstein College of Medicine, Bronx, NY, USA

3 Radiology Division, Montefiore Medical Center, Bronx, NY, USA

4 Cedars-Sinai Medical Center, Los Angeles, CA, USA been suggested that the thrombotic diathesis associated with COVID-19 reflects endothelial cell dysfunction [2]. The severity of COVID-19 is characterized by a pro-inflammatory state [3]. Recent epidemiological studies have demonstrated that common cardiovascular risk factors, including hypertension, diabetes, chronic kidney disease and obesity are strongly associated with adverse outcomes in COVID-19 $[4,5]$ with patients from ethnic minorities at a particular risk [5]. Computed tomographic (CT) scanning can be used to detect and quantify visceral fat. In the past few years, epicardial adipose tissue (EAT) has attracted a lot of interest as a marker of cardiometabolic risk as an independent marker for coronary atherosclerosis development and progression [6]. EAT is a metabolically active fat depot that makes up for approximately $20 \%$ of total heart weight, lies between serous epicardium and the fibrous pericardium, and can be quantified from non-gated non-contrast chest CT [7]. EAT is strongly associated with subclinical atherosclerosis, inflammation and major cardiac events in asymptomatic subjects 
[8]. EAT can influence cardiomyocytes and cardiac function via a secretion of proinflammatory adipokines. CT scanning can also identify the presence and extent of coronary atherosclerosis. The coronary artery calcification scoring (CAC) is an established and extensively validated imaging biomarker for cardiovascular risk, allowing enhanced risk reclassification for the prediction of all cause and cardiac specific mortality in asymptomatic subjects [9]. CAC has demonstrated to be a better predictor of cardiovascular and all-cause mortality across the spectrum of traditional risk factors [10]. Although the traditional Agatston method for measuring CAC requires ECG-gated acquisition, good correlation has been demonstrated between CAC identified on non-gated CT scans and ordinal scores obtained from gated CT scans [11]. Our hypothesis was that presence of EAT and/or CAC, as markers of a pro-inflammatory milieu and coronary atherosclerosis, respectively, are independently associated with increased risk for death in COVID-19.

\section{Methods}

We conducted a retrospective, post-hoc analysis of all patients who were admitted to Montefiore Medical Center with a confirmed COVID-19 diagnosis by Polymerase Chain Reaction (PCR) from March 1st, 2020 to June 23rd, 2020. Sample for RT-PCR was obtained by either nasopharyngeal or oropharyngeal swab. The follow up period was from March 1st, 2020 to June 26th, 2020. Patients who had a noncontrast CT of the chest at our facility after March 1st, 2015 constituted the main study cohort. Baseline demographic, clinical and laboratory variables (including inflammatory biomarkers) were retrieved from our electronic medical record system. Baseline characteristics and outcomes were compared with patients who were admitted with COVID-19 but did not have a CT study performed. The investigators had direct access to primary data.

The study was approved by our Institutional Review Board (Office of Human Research Affairs at Albert Einstein College of Medicine).

\section{CT acquisition}

Patients were imaged on the following CT scanners without contrast administration or electrocardiographic gating: GE Optima 660, GE Lightspeed VCT, Neurologica Corp Caretom, Siemens Somatom Sensation 16 and GE Optima CT 540. Images were reconstructed at $2.5 \mathrm{~mm}$ slice thickness. Scans with reconstructions at different slice thickness were excluded.

\section{Coronary calcium score (CAC)}

Two readers blinded from clinical data calculated ordinal scores from standard non-gated chest CT studies using the methods described by Shemesh et al. for patients without history of PCI or CABG [11]. Briefly, the right coronary artery, left main, left anterior descending, and left circumflex arteries were each given a score of $\mathbf{0 - 3}$ for presence and extent of calcium with 0 (none), 1 ( $<1 / 3$ of the artery length calcified), 2 ( $\geq 1 / 3$ to $<2 / 3$ calcified), and 3 ( $\geq 2 / 3$ calcified). These scores were summed across the 4 arteries, providing a range of possible scores from 0 to 12 (See Fig. 1a and b). We calculated the inter-observer variability based on a random sample. Agreement for severity class was high (Interclass Correlation Coefficient $=0.980, \mathrm{p}<0.001$ ).

\section{Quantification of EAT and TAT}

For the measurement of EAT, we used QFAT software. As previously described [12], QFAT uses convolutional deep learning for fully automated quantification of epicardial and thoracic volumes. Accuracy and reproducibility of QFAT have been previously validated $[13,14]$. Contours were reviewed by an expert user and manually modified when needed. Superior and inferior limits of the pericardium were identified as the bifurcation of the pulmonary trunk and the posterior descending artery respectively. EAT was defined as adipose tissue in the pericardium and TAT as the fat in the mediastinum within the abovementioned parameters but
Fig. 1 CAC, EAT and TAT. a Patient with $\mathrm{CAC}=0$ who survived. b Patient with $\mathrm{CAC}=9$ who died. $\mathbf{c}$ Patient with $\mathrm{EAT}=138 \mathrm{ml}$ and TAT $=130 \mathrm{ml}$ who died; EAT highlighted in purple by QFAT Software

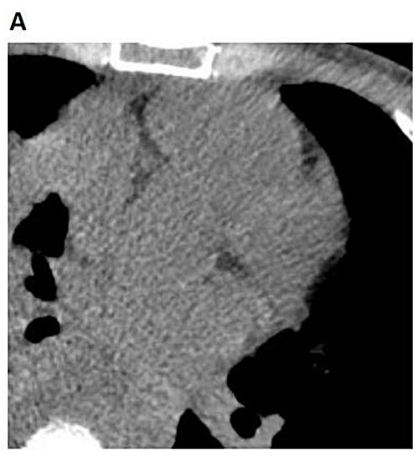

B

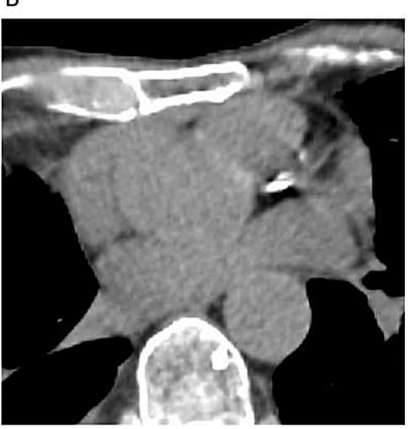

C

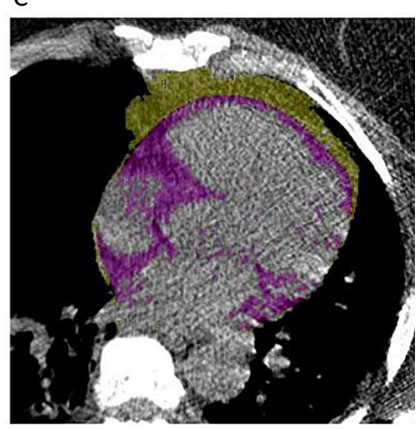


outside the pericardium (See Fig. 1c). Patients with significant artifacts from ICDs or poor acquisition were excluded.

\section{Statistical analysis}

Continuous data are displayed as mean \pm standard deviation or median [25-75\% interquartile range] and compared with the Student's t-test, or Wilcoxon ranks-sum, as appropriate. Categorical data are presented as percent and compared by the chi-squared test. As hospital discharge is a competing event with in-hospital mortality, instead of a Cox regression model, we used a proportional hazards model for the subdistribution of a competing risk analysis adjusting for covariates by Fine and Gray's method [15-17] to estimate the difference in the cumulative incidence of the outcome. June 26th, 2020 was the end of the censoring period. Intraclass correlation coefficient was calculated to determine interrater agreement. Pearson's product-moment correlation was utilized to assess the relationship between variables. A p $<0.05$ was considered statistically significant. Statistical analysis was performed with SPSS (IBM Corp, ver. 25, Armonk, $\mathrm{NY}$ ) and the R v3.3 package CMPRSK (R Foundation for Statistical Computing, ver 3.3, Vienna, Austria).

\section{Results}

\section{Study population and baseline characteristics.}

From 4311 patients who were hospitalized at Montefiore between March 1st and June 23rd, 2020 with confirmed COVID-19 by PCR, we identified 504 patients who had a non-contrast chest $\mathrm{CT}$ performed during or within 5 years prior to admission. 11 patients were excluded due to PCI, CABG or artifact. 493 patients were finally included for the clinical outcomes (455 for CAC and 457 for QFAT analysis). Overall median age was 70 (IQR 60-77) years, $49.5 \%$ were male and 33.3\% Hispanic. Diabetes (DM) (62.3\%), hyperlipidemia (74.6\%), hypertension (88.6\%) and asthma/COPD (52.3\%) were highly prevalent. The overall Charlson comorbidity index was 7 (IQR 4-10). On average patients presented to the hospital 2 days after onset of symptoms (IQR $0-5)$. There were 197 deaths with an in-hospital mortality of $40.0 \%$. Amongst traditional risk factors, patients who died were older (72, IQR 64-80 vs 68, IRQ 57-76 years; $\mathrm{p}<0.001)$, more likely to have history of CAD (54.8 vs $44.3 \% ; \mathrm{p}=0.021)$ and a higher Charlson comorbidity index (8, IQR 5-11 vs 7, IQR 4-10; $p=0.004)$. See Table 1 . There was no significant difference in body-mass index (27.5, IQR $22.1-31.9$ vs 27.2 , IQR $\left.23.9-32.0 \mathrm{~kg} / \mathrm{m}^{2} ; \mathrm{p}=0.314\right)$, DM (64.5 vs $60.8 \% ; \mathrm{p}=0.412$ ), hypertension ( 89.8 vs $87.8 \%$; $\mathrm{p}=0.491)$, hyperlipidemia $(75.6$ vs $74.0 \% ; \mathrm{p}=0.680)$ or asthma/COPD (52.3 vs $52.4 \%$; $\mathrm{p}=0.986)$.
On presentation, patients who died had higher heart rate (97, IQR $85-112$ vs 93, IQR 79-105 beats per minute; $\mathrm{p}=0.009)$, respiratory rate $(20, \mathrm{IQR} 18-25$ vs $19, \mathrm{IQR}$ $18-20$ breaths per minute; $\mathrm{p}<0.001)$ and lower oxygen saturation (94, IQR 88-98 vs 96, IQR 93-98\%; p < 0.001). There were no differences on days between onset of symptoms and hospital admission, temperature, systolic blood pressure or diastolic blood pressure.

Laboratory values on admission showed that patients who died had higher white blood count (8.2, IQR 5.6-12.9 vs 6.9, IQR 4.7-9.5; $\mathrm{p}<0.001)$, lower hemoglobin (11.3, IQR 9.9-13.6 vs 12.2 , IQR 10.4-13.4; $\mathrm{p}<0.001$ ), lower estimated eGFR (31, IQR 15-61 vs 58, IQR 31-81; p <0.001) and higher of the following parameters: pro-BNP $(3,048$, IQR 765-15,000 vs 637, IQR 150-2,527; p <0.001), glucose (138, IQR 112-2058; 126, IQR 102-174; $p=0.014)$, d-dimer (2.8, IQR 1.3-5.4 vs 1.8, IQR 0.9-3.8; $\mathrm{p}=0.023$ ), C-reactive protein (14.2, IQR 7.7-24.8 vs 8, IQR 2.7-16.3; $\mathrm{p}<0.001)$, LDH (451, IQR 306-610 vs 329, IQR 266-426; $\mathrm{p}<0.001$ ), ferritin (1062, IQR 370-2717 vs 537, IQR 280-1256; $\mathrm{p}=0.008)$, pro-calcitonin $(0.8$, IQR $0.3-5.4$ vs 0.2 , IQR $0.1-0.9 ; \mathrm{p}<0.001)$ and troponin $(0.03$, IQR $0.01-0.09$ vs 0.01 , IQR $0.01-0.03 ; \mathrm{p}<0.001$ ).

Patients who died were more likely to receive antibiotics (90.9 vs 74.7\%; $\mathrm{p}<0.001$ ), statins (39.6 vs $16.9 \%$; $\mathrm{p}<0.001$ ) and less likely to be on angiotensin-receptor blocking agents ( 36.0 vs 45.6; $p=0.035$ ). There was no difference in the use of hydroxychloroquine ( 67.5 vs $63.5 \%$; $\mathrm{p}=0.361$ ), azithromycin (25.4 vs $28.0 \%$; $\mathrm{p}=0.515)$ or intravenous steroids $(6.1$ vs $10.5 \% ; \mathrm{p}=0.091$ ) administration between the groups. History of CAD mildly correlated with the presence of CAC $\geq 1$ $(r=0.331, p<0.001) .53 .9 \%$ of patients without history of CAD had CAC $\geq 1$ and $15.0 \%$ of patients with history of $\mathrm{CAD}$ had $\mathrm{CAC}=0$. As predictor of mortality, history of CAD had an AUC of 0.551 with $p=0.075$.

When compared to the group admitted for COVID19 within the same timeframe without a prior CT within 5 years $(\mathrm{n}=3,807)$, patients with no CT were younger ( 65 [54-77] vs 70 [60-77]; $\mathrm{p}<0.001)$, had higher BMI (28.8 [24.9-33.9] vs 27.3 [23.3-31.9]; p p <0.001), had less DM (51.9 vs $62.3 \%$; $\mathrm{p}<0.001$ ), hyperlipidemia ( 55.0 vs $74.6 \%$; $\mathrm{p}<0.001)$, hypertension (69.5 vs $88.6 \%, \mathrm{p}<0.001)$, CAD (23 vs $48.5 \%, \mathrm{p}<0.001)$ and lung disease $(25.2$ vs $52.3 \%$, $\mathrm{p}<0.001)$. There was no difference in gender $(53.2 \mathrm{vs}$ $49.5 \%, p=0.12$ ). Mortality for the cohort without prior CT was lower than one with prior CT ( 28.1 vs $40.0 \%, \mathrm{p}<0.001)$.

\section{Coronary artery calcium}

Ordinal CAC score was calculated in 455 patients. CAC $>0$ was present in 308 patients with 153 patients having mild (1-3), 94 moderate (4-6) and 61 severe (7-12) CAC with an overall population median CAC score of 2 
Table 1 Baseline characteristics of patients admitted with COVID-19

$\begin{array}{llll}\text { All } & \text { Alive } & \text { Dead } & p \\ \mathrm{n}=493 & \mathrm{n}=296 & \mathrm{n}=197\end{array}$

\begin{tabular}{|c|c|c|c|c|c|}
\hline \multicolumn{6}{|l|}{ Demographics } \\
\hline Age, year & $\mathrm{n}=493$ & $70(60-77)$ & $68(57-76)$ & $72(64-80)$ & $<0.001$ \\
\hline BMI $\left(\mathrm{kg} / \mathrm{m}^{2}\right)$ & $\mathrm{n}=482$ & $27.3(23.3-31.9)$ & $27.2(23.9-32)$ & $27.5(22.1-31.9)$ & 0.314 \\
\hline Male gender, no (\%) & $\mathrm{n}=493$ & $244 / 493(49.5)$ & $148 / 296(50)$ & $96 / 197$ (48.7) & 0.783 \\
\hline \multicolumn{6}{|l|}{ PMH } \\
\hline Diabetes, no (\%) & $\mathrm{n}=493$ & $307 / 493$ (62.3) & $180 / 296(60.8)$ & 127/197 (64.5) & 0.412 \\
\hline Hyperlipidemia, no (\%) & $\mathrm{n}=493$ & $368 / 493(74.6)$ & $219 / 296(74)$ & 149/197 (75.6) & 0.680 \\
\hline Hypertension, no (\%) & $\mathrm{n}=493$ & $437 / 493(88.6)$ & $260 / 296(87.8)$ & $177 / 197(89.8)$ & 0.491 \\
\hline Asthma/COPD, no (\%) & $\mathrm{n}=493$ & $258 / 493(52.3)$ & $155 / 296(52.4)$ & $103 / 197(52.3)$ & 0.986 \\
\hline Coronary artery disease (CAD) & $\mathrm{n}=493$ & $296 / 493(60.0)$ & $131 / 296(44.3)$ & $108 / 197(54.8)$ & 0.021 \\
\hline Charlson Score & $\mathrm{n}=493$ & $7(4-10)$ & $7(4-10)$ & $8(5-11)$ & 0.004 \\
\hline \multicolumn{6}{|l|}{ Presentation } \\
\hline Symptom duration, days & $\mathrm{n}=476$ & $2(0-5)$ & $2(0-5)$ & $2(0-4)$ & 0.073 \\
\hline Temperature, F & $\mathrm{n}=489$ & $98.6(98.0-99.7)$ & $98.6(98.0-99.6)$ & $98.7(98.0-99.8)$ & 0.421 \\
\hline Systolic BP, mmHg & $\mathrm{n}=492$ & $131(111-148)$ & $133(114-149)$ & $128(107-147)$ & 0.085 \\
\hline Diastolic BP, mmHg & $\mathrm{n}=490$ & $73(62-83)$ & $73(63.5-84)$ & $72(58.5-82)$ & 0.084 \\
\hline $\mathrm{HR}, \mathrm{bpm}$ & $\mathrm{n}=491$ & $95(81-109)$ & $93(79-105)$ & $97(85-112)$ & 0.009 \\
\hline Pulse oximeter saturation, $\%$ & $\mathrm{n}=490$ & $95(91-98)$ & $96(93-98)$ & $94(88-98)$ & $<0.001$ \\
\hline Respiratory rate, bpm & $\mathrm{n}=490$ & $20(18-22)$ & $19(18-20)$ & $20(18-25)$ & $<0.001$ \\
\hline WBC count, $\mathrm{k} / \mu \mathrm{l}$ & $\mathrm{n}=482$ & $7.3(4.9-10.8)$ & $6.9(4.7-9.5)$ & $8.2(5.6-12.9)$ & $<0.001$ \\
\hline Hemoglobin, g/dl & $\mathrm{n}=482$ & $11.9(10.2-13.4)$ & $12.2(10.4-13.4)$ & $11.3(9.9-13.6)$ & 0.045 \\
\hline Sodium, mEq/1 & $\mathrm{n}=479$ & $137(134-141)$ & $137(133-141)$ & $138(134-143)$ & 0.022 \\
\hline EGFR, ml/min/BSA & $\mathrm{n}=481$ & $48(21-76)$ & $58(30-81)$ & $31(15-61)$ & $<0.001$ \\
\hline Glucose & $\mathrm{n}=481$ & $130(107-188)$ & $126(102-174)$ & $138(112-205)$ & 0.014 \\
\hline Lactic acid, mmol/l & $\mathrm{n}=431$ & $2(1.5-2.9)$ & $1.8(1.4-2.6)$ & $2.3(1.6-3.3)$ & $<0.001$ \\
\hline ProBNP, pg/ml & $\mathrm{n}=263$ & $1223(227-5751)$ & $637(150-2527)$ & $3049(765-15,000)$ & $<0.001$ \\
\hline D-dimer, $\mu \mathrm{g} / \mathrm{ml}$ & $\mathrm{n}=199$ & $2.1(1-4.4)$ & $1.8(0.9-3.8)$ & $2.8(1.3-5.4)$ & $\mathbf{0 . 0 2 3}$ \\
\hline C-reactive protein, $\mu \mathrm{g} / \mathrm{ml}$ & $\mathrm{n}=229$ & $10.3(4.1-19.2)$ & $8(2.7-16.3)$ & $14.2(7.7-24.8)$ & $<0.001$ \\
\hline Fibrinogen, mg/dl & $\mathrm{n}=142$ & $634(492-744)$ & $622(471-735)$ & $645(521-762)$ & 0.148 \\
\hline LDH, U/l & $\mathrm{n}=299$ & $364(278-490)$ & $329(266-426)$ & $451(306-610)$ & $<0.001$ \\
\hline Ferritin, ng/ml & $\mathrm{n}=178$ & $656(309.3-1771)$ & $537(280-1256)$ & $1062(340-2717)$ & 0.008 \\
\hline Troponin $\mathrm{T}, \mathrm{ng} / \mathrm{ml}$ & $\mathrm{n}=406$ & $0.01(0.01-0.06)$ & $0.01(0.01-0.03)$ & $0.03(0.01-0.09)$ & $<0.001$ \\
\hline \multicolumn{6}{|l|}{ Medications during admission } \\
\hline Hydroxychloroquine, no (\%) & $\mathrm{n}=493$ & $321 / 493(65.1)$ & $188 / 296(63.5)$ & 133/197 (67.5) & 0.361 \\
\hline Azithromycin, no (\%) & $\mathrm{n}=493$ & $133 / 493(27)$ & $83 / 296(28)$ & $50 / 197(25.4)$ & 0.515 \\
\hline Other antibiotics, no (\%) & $\mathrm{n}=493$ & $400 / 493(81.1)$ & $221 / 296(74.7)$ & 179/197 (90.9) & $<0.001$ \\
\hline IV steroids, no (\%) & $\mathrm{n}=493$ & $43 / 493(8.7)$ & $31 / 296(10.5)$ & $12 / 197(6.1)$ & 0.091 \\
\hline ACE, no (\%) & $\mathrm{n}=493$ & $25 / 493(5.1)$ & $16 / 296(5.4)$ & 9/197 (4.6) & 0.678 \\
\hline ARBS, no (\%) & $\mathrm{n}=493$ & 206/493 (41.8) & $135 / 296(45.6)$ & 71/197 (36) & $\mathbf{0 . 0 3 5}$ \\
\hline Statin, no (\%) & $\mathrm{n}=493$ & $128 / 493(26)$ & $50 / 296(16.9)$ & 78/197 (39.6) & $<0.001$ \\
\hline \multicolumn{6}{|l|}{$\mathrm{CAC}$ and EAT } \\
\hline $\mathrm{CAC} \geq 1$ & $\mathrm{n}=455$ & $308 / 455(67.7)$ & $177 / 274(64.6)$ & $131 / 181(72.4)$ & 0.08 \\
\hline CAC & $\mathrm{n}=455$ & $2(0-5)$ & $1(0-4)$ & $3(0-6)$ & $<0.001$ \\
\hline Epicardial fat, ml & $\mathrm{n}=457$ & $98(67-141)$ & $94(64-129)$ & $107(70-152)$ & 0.023 \\
\hline Thoracic fat, ml & $\mathrm{n}=457$ & $174(111-270)$ & $169(108-252)$ & $187(115-291)$ & 0.061 \\
\hline
\end{tabular}

Columns show the different demographic, clinical, laboratory and CT values of the overall group (All), those who survived (Alive) and those who died (Dead). P value compared alive vs dead groups

$A L T$ Alanine transaminase; AST Aspartate transaminase; BMI Body mass index; BP Blood pressure; $B U N$ Blood urinary nitrogen; $C A C$ Ordinal Coronary artery calcium; $C A D$ Coronary artery disease; $C O P D$ Chronic obstructive pulmonary disease; $E G F R$ Estimated glomerular filtration rate; $F$ Fahrenheit; $H R$ Heart rate; $I L-6$ Interleukin 6; IV Intravenous; $L D H$ Lactate dehydrogenase; proBNP ProB-type natriuretic peptide; $W B C$ White blood cell 
(IQR 0-5). Patients who died had higher ordinal CAC score (3, IQR 0-6 vs 1, IQR 0-4; p $<0.001$; Fig. 2). Patients with $\mathrm{CAC} \geq 4$ were older (76.0 [68.0-82.0] vs 66 [56.0-74.8]; $\mathrm{p}<0.001)$, with lower BMI $(25.8$ [22.8-31.0] vs 28.2 [23.9-32.5]; $\mathrm{p}<0.001)$, higher percentage hyperlipidemia $(81.9$ vs $68.7 \%, \mathrm{p}<0.001)$, and CAD (61.3 vs $35.5 \%, \mathrm{p}<0.001)$. There was no difference on gender (male 52.9 vs $45.0 \%, \mathrm{p}=0.11$ ), DM (67.1 vs $58.7 \%, \mathrm{p}=0.08$ ), hypertension ( 91.6 vs $86.0 \%, \mathrm{p}=0.08$ ) or lung disease ( 54.8 vs $50.3 \%, \mathrm{p}=0.36)$. When adjusted for age, BMI, DM, HLD and HTN, CAC remained an independent risk factor for in-hospital mortality (OR 1.082, $\mathrm{p}=0.005$ ). For each point increase in CAC, mortality increased by $8 \%$. On a ROC curve analysis CAC showed an AUC of $0.602(\mathrm{p}<0.001)$. A CAC $\geq 4$ was associated with a crude mortality of 53\% (OR 1.634. See Fig. 3A; $\mathrm{p}=0.003)$. CAC $\geq 4$ remained as an independent predictor of mortality when adjusted for Charlson index (OR 1.818, $\mathrm{p}<0.001$ ). Additionally, ASCVD risk by the pooled cohort equation was not a good predictor of COVID-19 death (AUC for ASCVD 0.538, $\mathrm{p}=0.439$ ).

\section{Epicardial adipose tissue}

EAT and TAT were calculated in 457 patients. Median volume in the overall population was $98 \mathrm{ml}(67-141)$ and $174 \mathrm{ml}$ (111-270) for EAT and TAT, respectively. Patients who died had higher EAT volume (See Fig. 3b; $107 \mathrm{ml}$, IQ 70-152 vs 94 ml, IQ 64-129; $p=0.023$ ). On a ROC curve analysis EAT showed an AUC of $0.565(p=0.024)$. In contrast, TAT volumes did not differ between those who died and those who survived (187 ml, IQ 115-291 vs $169 \mathrm{ml}$, IQ $108-252 ; p=0.061)$. Patients with EAT over the median were older (71.0 [63.0-79.0] vs 65.5 [56.8-76.0]; $\mathrm{p}<0.001$, with higher BMI (28.6 [25.2-33.3] vs 25.4 [21.3-30.1]; $\mathrm{p}<0.001)$, higher percentage of hyperlipidemia (83.8 vs $64.4 \%, \mathrm{p}<0.001)$, DM (68.5 vs $57.2 \%, \mathrm{p}=0.01)$, hypertension (93.2 vs $84.7 \%, \mathrm{p}=0.004)$, CAD (55.3 vs $38.3 \%$,
Fig. 2 Mortality according to CAC severity. Bars show the median and interquartile range of CT patients with different CAC severity categories as per Shemesh et al. [11]. Patients with intermediate (CAC 4-6; $\mathrm{n}=94)$ and high (CAC 7-12; $\mathrm{n}=61$ ) coronary calcification had significantly increased mortality when compared to ones without $(\mathrm{CAC} 0 ; \mathrm{n}=147)$ or with low $(1-3 ; n=153)$ calcification. (CAC $4-6$ and $\mathrm{CAC}$ $7-12$ vs CAC 0 and CAC $1-3$; $\mathrm{p}<0.01)$

Fig. 3 Mortality in relation to CAC $\geq 4$ and $E A T \geq$ Median. Curves show cumulative incidence of death in days according to CAC score (a) and $\operatorname{EAT}(\mathbf{b})$
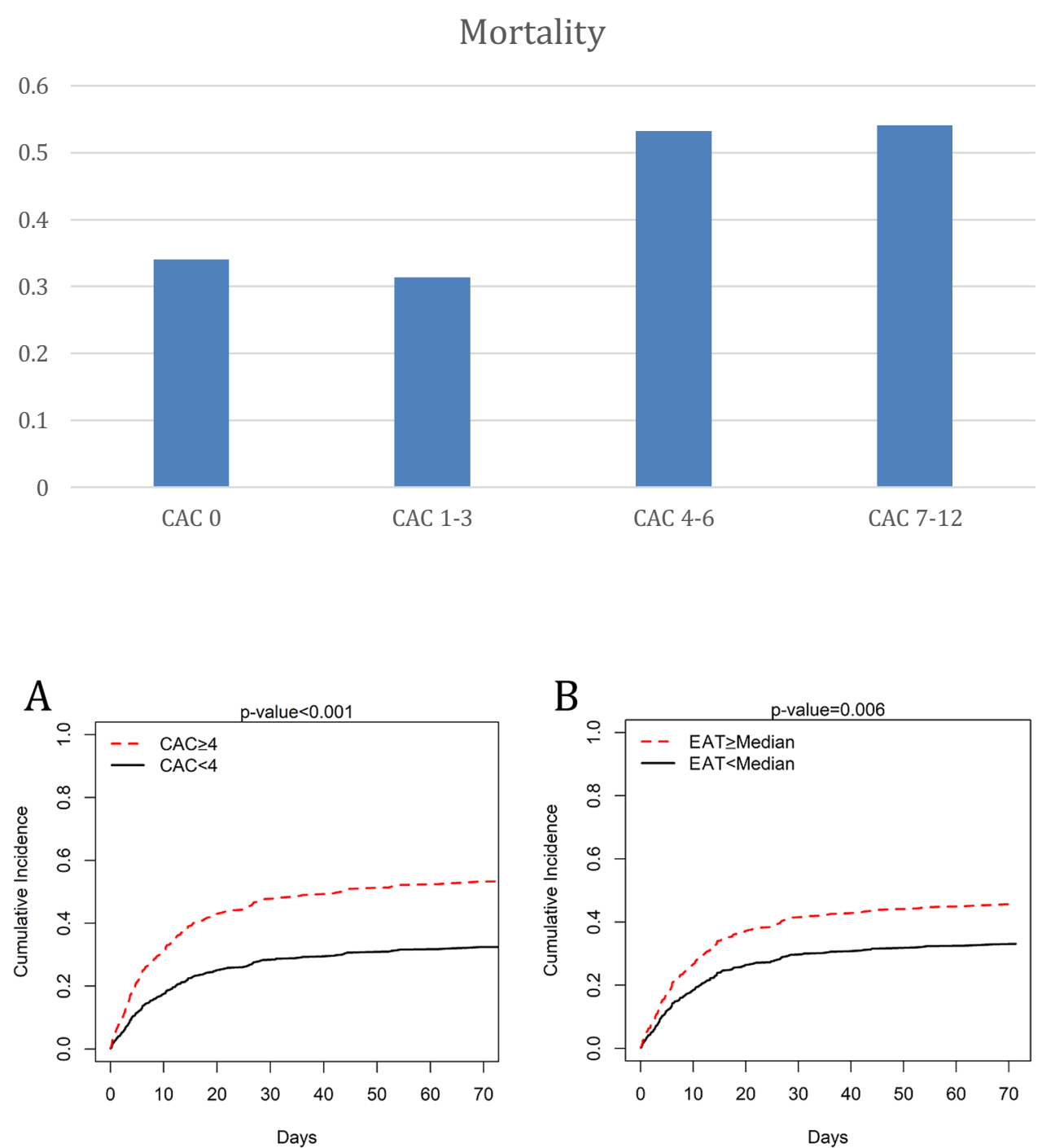
$\mathrm{p}<0.001)$ and lung disease (57.4 vs $46.4, \mathrm{p}=0.02)$. There was no difference on gender between the groups. There was a mild correlation between BMI and EAT $(r=0.33, p<0.001)$. When adjusted for age, BMI, DM, HLD and HTN, an EAT value above the median remained an independent risk factor for in-hospital mortality (crude mortality 46\%; OR 1.435, $\mathrm{p}=0.032$ ). EAT $\geq$ median remained as an independent predictor of mortality when adjusted for Charlson index (OR $1.534, \mathrm{p}=0.007)$.

When taken together, patients with $\mathrm{CAC} \geq 4$ and EAT $\geq$ median had the highest in-hospital mortality, See Fig. 4. CAC $<4$ and EAT $<$ median $(26 \%, 35 / 134)$; CAC $<4$ and EAT $\geq$ median $(28 \%, 54 / 141) ; \mathrm{CAC} \geq 4$ and EAT $<$ median $(46 \%, 31 / 68)$ and $\mathrm{CAC} \geq 4$ and $\mathrm{EAT} \geq$ median $(59 \%, 45 / 76), \mathrm{p}<0.001$.

\section{Discussion}

The main finding in our study is the independent association of CAC and EAT with all-cause mortality in a large cohort of patients admitted with COVID-19. This association was observed even after correcting for traditional risk factors that have been linked to worse COVID-19 outcomes such as age, BMI, diabetes, hypertension and hyperlipidemia.

To our knowledge, this represents the first study to show an independent association of CAC score and EAT with in-hospital mortality in confirmed COVID-19 patients. We showed that for each point increase in CAC, mortality increased by $8 \%$. On a regression model CAC $\geq 4$ independently increased mortality by $63 \%$ (Fig. 3a) and an EAT $\geq$ median $(98 \mathrm{ml}$ ) by $43 \%$. Importantly when taken in combination, the group with $\mathrm{CAC} \geq 4$ and $\mathrm{EAT} \geq$ median had highest mortality ( $<0.001$; Fig. 4$)$.

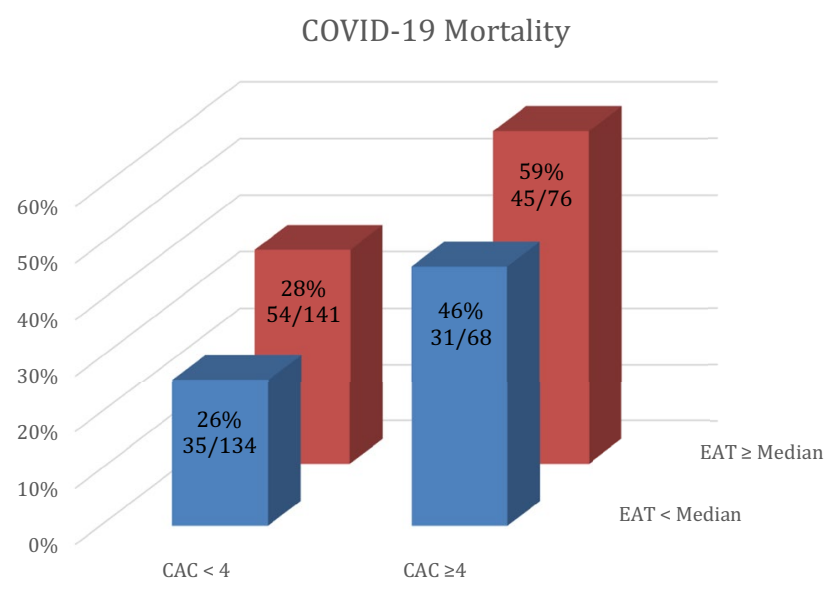

Fig. 4 Composite COVID-19 mortality for combination of CAC and EAT. Columns show percent of mortality for each group
It has been demonstrated that EAT is metabolically different from other visceral fat, both biochemically and in relation to cardiac risk factors. Multiple studies have postulated a prognostic role of EAT for clinical outcomes. Moreover, the role of EAT is incremental beyond CAC scoring [18]. Zhou et al. demonstrated that prediction of obstructive CAD was improved when EAT $>100 \mathrm{ml}$ (as a binary marker) was added to a model based on age, gender, angina and CAC in patients with stable angina [19]. Larsen et al. found a significant association of epicardial but not thoracic adipose tissue with earlier all-cause mortality in older adults over a 12-year follow up period [20].

Additionally, Gauss et al. found a significant association between CAC, EAT and systemic inflammatory markers [21]. Severity of COVID-19 infection has been associated to an immune dysregulation [22]. In particular, monocytes from COVID-19 patients have sustained expression of the cytokines tumor necrosis factor (TNF) and interleukin-6 (IL6) [23]. Mazurek et al. found that EAT is a source of several inflammatory mediators in high-risk cardiac patients with local expression of monocyte chemotactic protein (MCP)1 , IL-6 and TNF- $\alpha$ [24]. Other studies have also shown that EAT has prognostic value independently from clinical risk factors. One small study by Eslami et al. recently showed a relationship between EAT density and COVID-19 mortality but did not see an association with EAT thickness [25].

It is known that baseline pro-inflammatory risk factors (such as diabetes, hypertension, CAD and COPD) exacerbate the cytokine storm related to severe cases of COVID-19 [26]. We showed here that EAT, possibly through a proinflammatory local response has independent predictive value of all-cause mortality in COVID-19 [27].

Systemic inflammation adversely influences the biology of epicardial adipose tissue, promoting the expression of a proinflammatory phenotype. Furthermore, the release of pro-inflammatory adypocytokines from epicardial adipose tissue may contribute to the systemic inflammatory state as EAT appears to be a transducer that mediates the influence of systemic inflammation on adjacent tissues [28].

Measurement of EAT by CT has represented in the past a cumbersome process limiting its clinical applicability. Fully automated quantification by QFAT, through the use of a convolutional neural network approach, brings measurement process to a clinically useful and readily available tool with an average quantification time of $1.57 \mathrm{~s}$ [14]. Importantly, EAT quantification does not require ECG-gating nor additional radiation or acquisition time and the calculation through QFAT requires little to no training.

Data from multiple cohorts shows that CAC effectively stratifies patients for risk of long-term all-cause and cardiovascular mortality better than traditional risk-factors [9, $10,29,30]$. On the contrary, the effects of CAC on hospital mortality due to sepsis have been less explored. In one 
small study, Gupta et al. demonstrated that CAC stratifies septic patients for cardiovascular complications better than traditional risk factors [31]. CAC score was also evaluated in COVID-19 patients in smaller trials. A study from an Italian cohort of patients (332 patients, 68 deaths and mortality of $20.5 \%$ ) found a correlation between CAC on admission and mortality that did not persist after multivariate correction [32]. Compared to our study, patients in the study by Ferrante et al. had significantly lower comorbidities with less diabetes and dyslipidemia and lower incidence of CAC (CAC $\geq 1$ of $43.9 \%$ vs. $76.7 \%$ in our study) and a lower incidence of events, possibly explaining the different findings. Other small studies suggested a correlation of CAC and adverse events such as mechanical ventilation/ECMO or death [33-35]. Due to smaller sample size and lower event rate, these studies were hypothesis generating but could not confirm the premise.

Lastly, CAC $\geq 4$ and AET $\geq$ median had AUC of 0.596 $(p=0.001)$ and $0.565(p=0.024)$, respectively, whereas history of CAD had an AUC of 0.551 without statistical significance $(p=0.075)$ with a large percentage of patients having undiagnosed $\mathrm{CAC} \geq 1(53.9 \%)$ and $15.0 \%$ of patients with history of CAD but $\mathrm{CAC}=0$.

Our findings could have significant clinical implications on selection of higher risk patients for allocation of vaccines or admission beds in particular in countries with limited resources.

\section{Limitations}

The inclusion of only patients who had a prior chest CT selected a higher risk population, reflected in the higher mortality rate observed on patients with prior CT. Moreover, CTs were obtained up to 5 years before index hospitalization. Even that progression of coronary calcification within 5 years tends to be mild and within a severity class, we cannot exclude higher variation is some of our patient's population. However, we believe it is extremely important to have risk stratifying tools in sicker patients with higher risk for adverse events. Many inflammatory markers were not routinely collected at the beginning of the pandemic and we cannot therefore evaluate their interaction. Smoking is known to worsen COVID-19 outcomes, but smoking status could not be consistently ascertained. In our study, EAT showed only a modest additive value to $\mathrm{CAC}$ for predicting mortality. Co-linearity between variables and the high percentage of $\mathrm{CAC}$ in our population are possible explanations for this finding. Hypothetically, in a younger population EAT could have shown a larger additive value.

Finally, our cohort was selected from the initial wave of patients admitted to the hospital. Since the onset of the COVID-19 pandemic, treatment has evolved, and mortality of hospitalized patients has dramatically decreased. Thus, our results should be prospectively confirmed.

\section{Conclusion}

CAC and EAT measured by non-contrast chest $\mathrm{CT}$ are independent predictors of in-patient COVID-19 mortality in this high-risk cohort.

Author contributions LS: Conceptualization, Methodology, Validation, Formal Analysis, Investigation, Resources, Data Curation, WritingOriginal Draft, Visualization, Supervision; FC: Conceptualization, Methodology, Validation, Formal Analysis, Investigation, Data Curation, Writing-Review \& Editing; AS: Investigation; EN: Investigation; RS: Investigation; DD: Software, Writing-Review \& Editing, Funding Acquisition; UPJ: Conceptualization, Writing-Review \& Editing; JML: Conceptualization, Methodology, Validation, Resources, Data Curation, Investigation; MJG: Conceptualization, Methodology, Validation, Formal Analysis, Investigation, Resources, Data Curation, WritingReview \& Editing, Visualization, Supervision, Project Administration.

Funding Dr. Dey is partly supported by NIH/NHLBI Grant R01HL133616.

\section{Declarations}

Conflict of interest The authors declare that they have no conflict of interest.

Ethical Approval All human studies have been approved by the appropriate ethics committee and have therefore been performed in accordance with the ethical standards laid down in the 1964 Declaration of Helsinki and its later amendments. The study was approved by our Institutional Review Board (Office of Human Research Affairs at Albert Einstein College of Medicine).

Consent to participate Consent requirement was waived as per IRB guidelines.

Consent for publications Consent requirement was waived as per IRB guidelines.

\section{References}

1. John Hopkins University (2021) COVID-19 Dashboard by the Center for Systems Science and Engineering (CSSE) at Johns Hopkins University (JHU)

2. Escher R, Breakey N, Lämmle B (2020) Severe COVID-19 infection associated with endothelial activation. Thromb Res 190:62

3. Velavan TP, Meyer CG (2020) Mild versus severe COVID-19: laboratory markers. Int J Infect Dis 95:304-307. https://doi.org/ 10.1016/j.jijid.2020.04.061

4. Guo T, Fan Y, Chen M et al (2020) Cardiovascular implications of fatal outcomes of patients with coronavirus disease 2019 (COVID-19). JAMA Cardiol. https://doi.org/10.1001/jamacardio. 2020.1017 
5. Tartof SY, Qian L, Hong V et al (2020) Obesity and mortality among patients diagnosed with COVID-19: results from an Integrated Health Care Organization. Ann Intern Med. https://doi.org/ 10.7326/m20-3742

6. Yerramasu A, Dey D, Venuraju S et al (2012) Increased volume of epicardial fat is an independent risk factor for accelerated progression of sub-clinical coronary atherosclerosis. Atherosclerosis. https://doi.org/10.1016/j.atherosclerosis.2011.09.041

7. Nagayama Y, Nakamura N, Itatani R et al (2019) Epicardial fat volume measured on nongated chest $\mathrm{CT}$ is a predictor of coronary artery disease. Eur Radiol. https://doi.org/10.1007/ s00330-019-06079-x

8. Goeller M, Achenbach S, Marwan M et al (2018) Epicardial adipose tissue density and volume are related to subclinical atherosclerosis, inflammation and major adverse cardiac events in asymptomatic subjects. J Cardiovasc Comput Tomogr. https://doi. org/10.1016/j.jcct.2017.11.007

9. Greenland P, LaBree L, Azen SP et al (2004) Coronary artery calcium score combined with Framingham score for risk prediction in asymptomatic individuals. J Am Med Assoc. https://doi. org/10.1001/jama.291.2.210

10. Nasir K, Rubin J, Blaha MJ et al (2012) Interplay of coronary artery calcification and traditional risk factors for the prediction of all-cause mortality in asymptomatic individuals. Circ Cardiovasc Imaging. https://doi.org/10.1161/CIRCIMAGING.111.964528

11. Shemesh J, Henschke CI, Shaham D et al (2010) Ordinal scoring of coronary artery calcifications on low-dose CT scans of the chest is predictive of death from cardiovascular disease. Radiology. https://doi.org/10.1148/radiol.10100383

12. Otaki Y, Rajani R, Cheng VY et al (2011) The relationship between epicardial fat volume and incident coronary artery calcium. J Cardiovasc Comput Tomogr. https://doi.org/10.1016/j.jcct. 2011.06.007

13. Nakazato R, Shmilovich H, Tamarappoo BK et al (2011) Interscan reproducibility of computer-aided epicardial and thoracic fat measurement from noncontrast cardiac CT. J Cardiovasc Comput Tomogr. https://doi.org/10.1016/j.jcct.2011.03.009

14. Commandeur F, Goeller M, Betancur J et al (2018) Deep learning for quantification of epicardial and thoracic adipose tissue from non-contrast CT. IEEE Trans Med Imaging. https://doi.org/10. 1109/TMI.2018.2804799

15. Gray RJ (1988) A class of K-sample tests for comparing the cumulative incidence of a competing risk. Ann Stat 8:1141-1154

16. Satagopan JM, Ben-Porat L, Berwick M et al (2004) A note on competing risks in survival data analysis. Br J Cancer 91:1229-1235

17. Fine JP, Gray RJ (1999) A proportional hazards model for the subdistribution of a competing risk. J Am Stat Assoc. https://doi. org/10.1080/01621459.1999.10474144

18. Spearman JV, Renker M, Schoepf UJ et al (2015) Prognostic value of epicardial fat volume measurements by computed tomography: a systematic review of the literature. Eur Radiol 25(11):33723381. https://doi.org/10.1007/s00330-015-3765-5

19. Zhou J, Chen Y, Zhang Y et al (2019) Epicardial fat volume improves the prediction of obstructive coronary artery disease above traditional risk factors and coronary calcium score. Circ Cardiovasc Imaging. https://doi.org/10.1161/CIRCIMAGING. 118.008002

20. Larsen BA, Laughlin GA, Saad SD et al (2015) Pericardial fat is associated with all-cause mortality but not incident CVD: The
Rancho Bernardo Study. Atherosclerosis. https://doi.org/10. 1016/j.atherosclerosis.2015.02.022

21. Gauss S, Klinghammer L, Steinhoff A et al (2015) Association of systemic inflammation with epicardial fat and coronary artery calcification. Inflamm Res. https://doi.org/10.1007/ s00011-015-0809-x

22. Yu K, He J, Wu Y et al (2020) Dysregulated adaptive immune response contributes to severe COVID-19. Cell Res 30:814-816

23. Giamarellos-Bourboulis EJ, Netea MG, Rovina N et al (2020) Complex immune dysregulation in COVID-19 patients with severe respiratory failure. Cell Host Microbe. https://doi.org/10. 1016/j.chom.2020.04.009

24. Mazurek T, Zhang LF, Zalewski A et al (2003) Human epicardial adipose tissue is a source of inflammatory mediators. Circulation. https://doi.org/10.1161/01.CIR.0000099542.57313.C5

25. Eslami V, Abrishami A, Zarei E et al (2020) The association of CT-measured cardiac indices with lung involvement and clinical outcome in patients with COVID-19. Acad Radiol. https://doi.org/ 10.1016/j.acra.2020.09.012

26. Huang C, Wang Y, Li X et al (2020) Clinical features of patients infected with 2019 novel coronavirus in Wuhan, China. Lancet. https://doi.org/10.1016/S0140-6736(20)30183-5

27. Malavazos AE, Goldberger JJ, Iacobellis G (2020) Does epicardial fat contribute to COVID-19 myocardial inflammation? Eur Heart J 41:2333. https://doi.org/10.1093/eurheartj/ehaa471

28. Packer M (2018) Epicardial adipose tissue may mediate deleterious effects of obesity and inflammation on the myocardium. J Am Coll Cardiol 71:2360-2372. https://doi.org/10.1016/j.jacc.2018. 03.509

29. Budoff MJ, Shaw LJ, Liu ST et al (2007) Long-term prognosis associated with coronary calcification observations from a registry of 25,253 patients. J Am Coll Cardiol. https://doi.org/10.1016/j. jacc.2006.10.079

30. Knapper JT, Khosa F, Blaha MJ et al (2016) Coronary calcium scoring for long-term mortality prediction in patients with and without a family history of coronary disease. Heart. https://doi. org/10.1136/heartjnl-2015-308429

31. Gupta VA, Sousa M, Kraitman N et al (2018) Coronary artery calcification predicts cardiovascular complications after sepsis. J Crit Care. https://doi.org/10.1016/j.jcrc.2017.11.038

32. Ferrante G, Fazzari F, Cozzi O et al (2020) Risk factors for myocardial injury and death in patients with COVID-19: insights from a cohort study with chest computed tomography. Cardiovasc Res. https://doi.org/10.1093/cvr/cvaa193

33. Matos J, Paparo F, Mussetto I et al (2020) Evaluation of novel coronavirus disease (COVID-19) using quantitative lung CT and clinical data: prediction of short-term outcome. Eur Radiol Exp. https://doi.org/10.1186/s41747-020-00167-0

34. Dillinger JG, Benmessaoud FA, Pezel T et al (2020) Coronary artery calcification and complications in patients with COVID-19. JACC Cardiovasc Imaging 13:2648

35. Nai Fovino L, Cademartiri F, Tarantini G (2020) Subclinical coronary artery disease in COVID-19 patients. Eur Heart J Cardiovasc Imaging 21:1055. https://doi.org/10.1093/ehjci/jeaa202

Publisher's Note Springer Nature remains neutral with regard to jurisdictional claims in published maps and institutional affiliations. 